\title{
Negative scattering asymmetry parameter for dipolar particles: Unusual reduction of the transport mean free path and radiation pressure
}

\author{
R. Gómez-Medina, ${ }^{1}$ L. S. Froufe-Pérez, ${ }^{1}$ M. Yépez, ${ }^{1}$ F. Scheffold,${ }^{2}$ M. Nieto-Vesperinas,,${ }^{3, *}$ and J. J. Sáenz ${ }^{1, \dagger}$ \\ ${ }^{1}$ Departamento de Física de la Materia Condensada and Instituto "Nicolás Cabrera," Universidad Autónoma de Madrid, \\ 28049 Madrid, Spain \\ ${ }^{2}$ Department of Physics, University of Fribourg, Chemin du Muse 3, 1700 Fribourg, Switzerland \\ ${ }^{3}$ Instituto de Ciencia de Materiales de Madrid, Consejo Superior de Investigaciones Cientificas, Campus de Cantoblanco, \\ Madrid 28049, Spain
}

(Received 31 January 2012; published 26 March 2012)

\begin{abstract}
Lossless dielectric nanospheres (made of nonmagnetic materials) with relatively low refraction index may present strong electric and magnetic dipolar resonances. We establish a relationship between the optical force from a plane wave on small electric and magnetic dipolar particles, the transport cross section, and the scattering asymmetry parameter $g$. In this way we predict negative $g$ (that minimize the transport mean free path below values of the scattering mean free path) for a dilute suspension of both perfectly reflecting spheres as well as of lossless dielectric nanospheres made of moderate permittivity materials, e.g., silicon or germanium nanospheres in the infrared region. Lossless dielectric Mie spheres of relatively low refraction index (as low as 2.2) are shown to present negative $g$ in specific spectral ranges.
\end{abstract}

DOI: 10.1103/PhysRevA.85.035802

PACS number(s): 42.25.Bs, 05.60.Cd, 42.25.Dd, 42.55.-f

Propagation of light and image formation in turbid media has long been a subject of great interest [1] and constitutes the core of powerful techniques with countless applications including biomedical imaging [2] and dynamic spectroscopy techniques [3], characterization of composite materials and complex fluids [4], remote sensing or telecommunications [5] to mention a few. Our current understanding of the diffusive transport through nonabsorbing media is based on the knowledge of two key quantities: the transport and scattering mean free paths (MFPs). The scatter density and cross section define the scattering MFP $\ell_{s}$. The relevant scattering length for diffusive light power transport is the transport MFP $\ell^{*}$. Both quantities are connected by the scattering asymmetry parameter $g$ defined [6,7] as the average of the cosine of the scattering angle $g \equiv\langle\cos \theta\rangle$ with $\ell^{*}=\frac{\ell_{s}}{1-g}$, where $\ell^{*}$ is usually equal to or larger than $\ell_{s}$, i.e., $g$ is positive. For instance, the isotropic Rayleigh scattering of small particles leads to $g \sim 0$ while Mie particles (or human tissue) [7] scatter strongly in the forward direction (small scattering angles) and hence $g \sim 1$. Negative values of $g$ were reported [8] for magnetic particles having electric permittivity $\epsilon>1$ and large values of the magnetic permeability $\mu \gg 1$. Nevertheless, no concrete example of such particles that might present $g<0$ in the visible or infrared regions has been proposed yet. However, recently it has been shown that subwavelength spheres made of nonabsorbing dielectric material with relatively low refractive index produce anisotropic angular distributions of scattered intensity [9-12]. As we will show here, these particles can present negative- $g$ values in specific wavelength regions, i.e., a random dispersion of such particles will show the unusual characteristic of having $\ell^{*}<\ell_{s}$, even in the absence of positional correlations.

\footnotetext{
*mnieto@icmm.csic.es

†juanjo.saenz@uam.es
}

The transport mean free path can be strongly modified by the presence of short-range structural order in the system $[13,14]$. Positional correlations usually lead to positive- $g$ values, i.e., to $\ell^{*}$ values significantly larger than $\ell_{s}$, which are responsible, for example, for the relatively large conductivity of disordered liquid metals [15] or the transparency of the cornea to visible light [16]. However, short-range order can also lead to negative values of the asymmetry parameter as it has been recently shown in experiments in colloidal liquids [17] and amorphous photonic materials [18]. These negative values, observed at specific wavelength regions, have been associated $[17,18]$ with enhanced backscattering at Bragg-like matching resonances [19].

The unusual observation of negative- $g$ factors has been limited to systems with appropriate short-range correlation between scatters. While it is frequently argued that the scattering from Mie spherical particles leads to $g>1$, in this work we show that nonabsorbing Mie spheres of relatively low refraction index $m$ present negative- $g$ factors in specific spectral ranges. Previous numerical work [20] reported calculated negative- $g$ factors for dielectric spheres having refractive index $m$ larger than 3.1. However, the physical origin of these results was not discussed. Interestingly, as we will see, they represent a specific example of small particles, whose scattering may be completely described by a dipolar response to both the electric and magnetic fields. In contrast, we show that relatively large, nondipolar, dielectric spheres may lead to $g<0$ for $m$ as low as 2.2.

As we will see, there is a close relationship between transport parameters of a dilute suspension of dipolar particles and the theory of optical forces on magnetodielectric small particles [10,11,21], in which it has been shown that, in addition to the force due to the electric and magnetic induced dipoles, there is an additional component due to the interaction between both of them, which was associated with the angular distribution of scattered intensity [10-12]. Maxima and (negative) minima of the $g$ factor are obtained for 
the so-called Kerker conditions [11,22,23] of zero-backward or almost zero-forward differential scattering cross sections (DSCSs). These conditions can be satisfied by hypothetical magnetic particles $[8,22,23]$ as well as by small dielectric particles of high refractive index (e.g., of $\mathrm{Si}$ or Ge), which have recently been shown to behave as magnetodielectric $[9,12]$, i.e., whose scattering is effectively dipolar, being well characterized by the Mie coefficients $a_{1}$ and $b_{1}$, both being of comparable strength. A dilute suspension of such particles near the almost-zero-forward scattering condition, will minimize the transport mean free path below the scattering mean free path.

The asymmetry factor $g=\langle\cos \theta\rangle$ is defined [6,7] as the average of the cosine of the scattering angle $\theta$ over the particle differential scattering-cross-section distribution $d \sigma_{s} / d \Omega$ :

$$
g=\langle\cos \theta\rangle=\frac{\int \frac{d \sigma_{s}}{d \Omega} \cos \theta d \Omega}{\int \frac{d \sigma_{s}}{d \Omega} d \Omega}=\frac{\int \frac{d \sigma_{s}}{d \Omega} \cos \theta d \Omega}{\sigma_{s}},
$$

where $\sigma_{s}$ is the scattering cross section. Let us compute the $g$ factor for a dielectric dipolar sphere of radius $a$ and real refractive index $m_{p}$ immersed in an arbitrary lossless medium with relative dielectric permittivity $\epsilon$ and magnetic permeability $\mu$. For spherical particles, the $g$ factor does not depend on the polarization of the incident light and can be expressed in terms of the Mie coefficients $a_{n}$ and $b_{n}$ (see Sec. 4.5 in Ref. [7]). In Fig. 1(a) we show the $g$ factor map for a nonabsorbing Mie sphere as a function of the relative refractive index $m=m_{p} / \sqrt{\epsilon \mu}$ and the size parameter $y \equiv m(2 \pi a / \lambda)$ calculated from the full Mie expansion. Since usually nonabsorbing materials present low refractive index $(m \lesssim 1.5)$ in the infrared and visible frequency ranges, negative- $g$ factors in Mie particles were not expected. However, as it can be seen in Fig. 1(a), the $g$ map shows regions of negative $g$ for relatively low refraction index $(m \gtrsim 2)$ relevant for semiconductor particles made of silicon $(m \approx 3.5)$ or germanium $(m \approx 4)$ in the infrared and telecom wavelengths. This is one of the main results of the present work. The corresponding scattering-cross-section map for the same spheres (as calculated in Ref. [9]) is plotted in Fig. 1(b). For $m$ values larger than approximately 2, the region between the magnetic [green in Fig. 1(b)] and electric [red in Fig. 1(b)] dipolar resonances presents a well-defined region of negative asymmetry parameter while the scattering is perfectly described by the first two dipolar terms in the Mie expansion [9].

The asymmetry factor $g$ and the seemingly unrelated problems of transport mean free path and optical forces are now tied together by the close relation between power and momentum transfer, i.e., by the definition of transport $\sigma^{*}$ and radiation pressure $\sigma^{(\mathrm{pr})}$ cross sections $[6,7,24]$. The transport cross section $\sigma^{*}$ of a particle is expressed in terms of $\sigma_{s}$ as $[1,3]$

$$
\sigma^{*}=\int \frac{d \sigma_{s}}{d \Omega}(1-\cos \theta) d \Omega=\sigma_{s}(1-g) .
$$

For a dilute suspension of optically uncorrelated particles with density $\rho$, the transport MFP $l^{*}=1 / \rho \sigma^{*}$ is related to the scattering MFP $l_{s}=1 / \rho \sigma_{s}$ through the aforementioned relationship $\ell^{*}=\frac{\ell_{s}}{1-g}$. In contrast, the radiation pressure

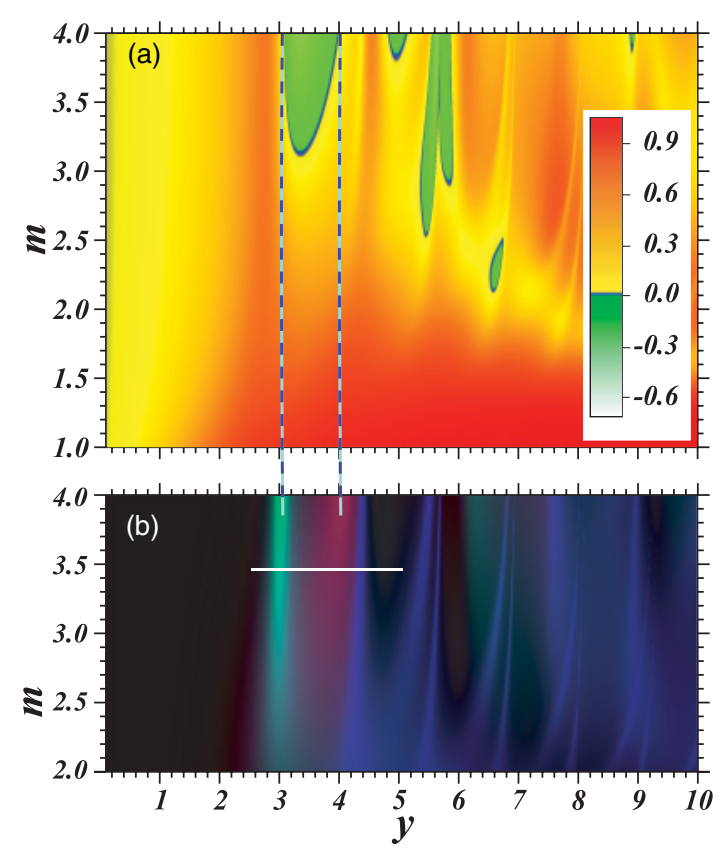

FIG. 1. (Color) (a) Color map of the $g$ factor for spherical absorptionless particles as a function of their refractive index $m$ and size parameter $y=m k a$. As seen in the attached scale, green areas correspond to negative values of $g$. (b) Color map of the sphere scattering cross section. Red corresponds to dominant electric dipole contributions to the scattering cross section. Green corresponds to dominant magnetic dipole contributions, while blue sums up all higher-order multipole terms. Vertical dashed lines coincide with $y$ parameter for maximum electric dipole contribution (right vertical line) and maximum magnetic dipole contribution (left vertical line). The white horizontal line at $m \approx 3.5$ (which corresponds to a silicon sphere) represents the $y$ range covered by Fig. 2 .

cross section is customarily defined as [6] $\sigma^{(\mathrm{pr})}=\sigma^{(\mathrm{ext})}-$ $\langle\cos \theta\rangle \sigma_{s}=\sigma_{a}+\sigma^{*}$, where $\sigma^{\text {(ext) }}=\sigma_{s}+\sigma_{a}$, with $\sigma_{a}$ being the absorption cross section. In the absence of absorption, there is no difference between transport and radiation pressure cross sections,

$$
\sigma^{*}=\sigma^{(\mathrm{pr})}=\sigma_{s}(1-g) .
$$

Hence there is a direct relation between transport quantities and the forces from an incident plane wave on a dielectric sphere.

In order to get deeper physical insight into the influence of the electric-magnetic dipole force in $\ell^{*}$, it is interesting to derive the explicit expressions connecting both transport and radiation pressure with the $g$ factor for the simplest and most important case of dipolar particles. Let us consider such a particle whose dipolar electric $\mathbf{p}$ and magnetic $\mathbf{m}$ moments are related to the external polarizing fields through $\mathbf{p}=\epsilon_{0} \epsilon \alpha_{e} \mathbf{E}$ and $\mathbf{m}=\left(\alpha_{m} / \mu_{0} \mu\right) \boldsymbol{B}$. The dynamic polarizabilities $\alpha_{e}$ and $\alpha_{m}$ that characterize the dipole excitation can be expressed in terms of the Mie coefficients $a_{1}$ and $b_{1}$ as [6,7] $\alpha_{e}=$ $i a_{1}\left(6 \pi / k^{3}\right)$ and $\alpha_{m}=i b_{1}\left(6 \pi / k^{3}\right)$ ( $k$ is the wave number $k=\sqrt{\epsilon \mu} \omega / c)$. 
The differential scattering cross section averaged over the incident polarizations is [10-12]

$$
\frac{d \sigma_{s}(\theta)}{d \Omega}=\frac{k^{4}}{16 \pi}\left(\left(\left|\alpha_{e}\right|^{2}+\left|\alpha_{m}\right|^{2}\right) \frac{1+\cos ^{2} \theta}{2}+2 \operatorname{Re}\left(\alpha_{e} \alpha_{m}^{*}\right) \cos \theta\right) .
$$

From Eqs. (4) and (1)

$$
g=\frac{\operatorname{Re}\left[\alpha_{e} \alpha_{\mathrm{m}}^{*}\right]}{\left|\alpha_{e}\right|^{2}+\left|\alpha_{m}\right|^{2}},
$$

which shows that for a dipolar particle, $|g| \leqslant 1 / 2$. Notice also that from Eqs. (4) and (5) this asymmetry factor may be expressed as

$$
g=\frac{1}{2}\left[\frac{\frac{d \sigma^{(s)}}{d \Omega}\left(0^{\circ}\right)-\frac{d \sigma^{(s)}}{d \Omega}\left(180^{\circ}\right)}{\frac{d \sigma^{(s)}}{d \Omega}\left(0^{\circ}\right)+\frac{d \sigma^{(s)}}{d \Omega}\left(180^{\circ}\right)}\right] .
$$

In the absence of absorption, the time-averaged force exerted on the dipolar particle by a time harmonic incident plane wave $\mathbf{E}=\mathbf{E}_{0} e^{i \mathbf{k} \cdot \mathbf{r}}$ is all radiation pressure $[10,11]$ and reads

$$
\begin{aligned}
\langle\mathbf{F}\rangle & =\left\langle\mathbf{F}_{e}\right\rangle+\left\langle\mathbf{F}_{m}\right\rangle+\left\langle\mathbf{F}_{e-m}\right\rangle \\
& =\frac{\epsilon_{0} \epsilon}{2}\left|\mathbf{E}_{0}\right|^{2}\left\{k \operatorname{Im}\left(\alpha_{e}+\alpha_{m}\right)-\frac{k^{4}}{6 \pi} \operatorname{Re}\left[\alpha_{e} \alpha_{m}^{*}\right]\right\} \\
& =\frac{\epsilon_{0} \epsilon}{2}\left|\mathbf{E}_{0}\right|^{2} \sigma^{(\mathrm{pr})} \frac{\mathbf{k}}{k} .
\end{aligned}
$$

The last term in Eq. (7), $\left\langle\mathbf{F}_{e-m}\right\rangle$, due to the interaction between electric and magnetic dipoles [10,11,21], was associated in Ref. [11] with the asymmetry in the scattered intensity distribution [cf. the last term in Eq. (4)] even though it was not explicitly related to $g$. We next show that they are proportional. Notice that the moduli of the first two terms $\left\langle\mathbf{F}_{e}\right\rangle$ and $\left\langle\mathbf{F}_{m}\right\rangle$, corresponding to the forces on the induced pure electric and magnetic dipoles, can be written as

$$
\left\langle F_{e}\right\rangle+\left\langle F_{m}\right\rangle=\frac{\epsilon_{0} \epsilon}{2}\left|\mathbf{E}_{0}\right|^{2} \sigma^{(\text {ext })}=\frac{\epsilon_{0} \epsilon}{2}\left|\mathbf{E}_{0}\right|^{2} \sigma_{s},
$$

where the last equality holds for nonabsorbing particles, while the interference term

$$
\left\langle F_{e-m}\right\rangle=-\frac{\epsilon_{0} \epsilon}{2}\left|\mathbf{E}_{0}\right|^{2} \sigma_{s} g .
$$

We then have a formal result for the total force

$$
\langle F\rangle=\left(\left\langle F_{e}\right\rangle+\left\langle F_{m}\right\rangle\right)(1-g),
$$

which is the force analog of Eq. (3). We can summarize the above discussion in a single expression

$$
1-g=\frac{\sigma^{*}}{\sigma_{s}}=\frac{\langle F\rangle}{\left\langle F_{e}\right\rangle+\left\langle F_{m}\right\rangle}=\frac{\ell_{s}}{\ell^{*}} .
$$

Equation (12) is another main result of this work. When the particle is nonabsorbing, $1-g$ becomes just the ratio between the magnitudes of the total force and the sum of the pure electric dipole forces. This quantifies in a specific way the nature of the interaction force component in terms of the forward-backward asymmetry of the angular distribution of scattered intensity by the particle. It also establishes the connection between these forces and the transport and scattering MFP's.
If no restrictions are imposed on $\alpha_{e}$ and $\alpha_{m}$, and hence one may consider them in Eq. (5) as independent variables, it is straightforward to see from this equation that $g$ takes on extreme values when either $\alpha_{e}=\alpha_{m}$ (with $g$ being a maximum $g=1 / 2$ ) or $\alpha_{e}=-\alpha_{m}$ (with $g$ being a minimum $g=-1 / 2$ ). The first condition corresponds to the so-called first Kerker condition and has been discussed in the context of scattering from a special case of magnetodielectric particles [11,22]. These particles lead to a zero-backward differential scattering cross section and have $g=1 / 2$, i.e., $l^{*}=2 l_{s}$ (notice that forward scattering would correspond to $g=1$ and $l^{(*)}=\infty$ ). The second condition $\left(\alpha_{e}=-\alpha_{m}\right)$, which minimizes the scattered intensity in the forward direction, can only be fulfilled approximately since the imaginary part of the polarizabilities

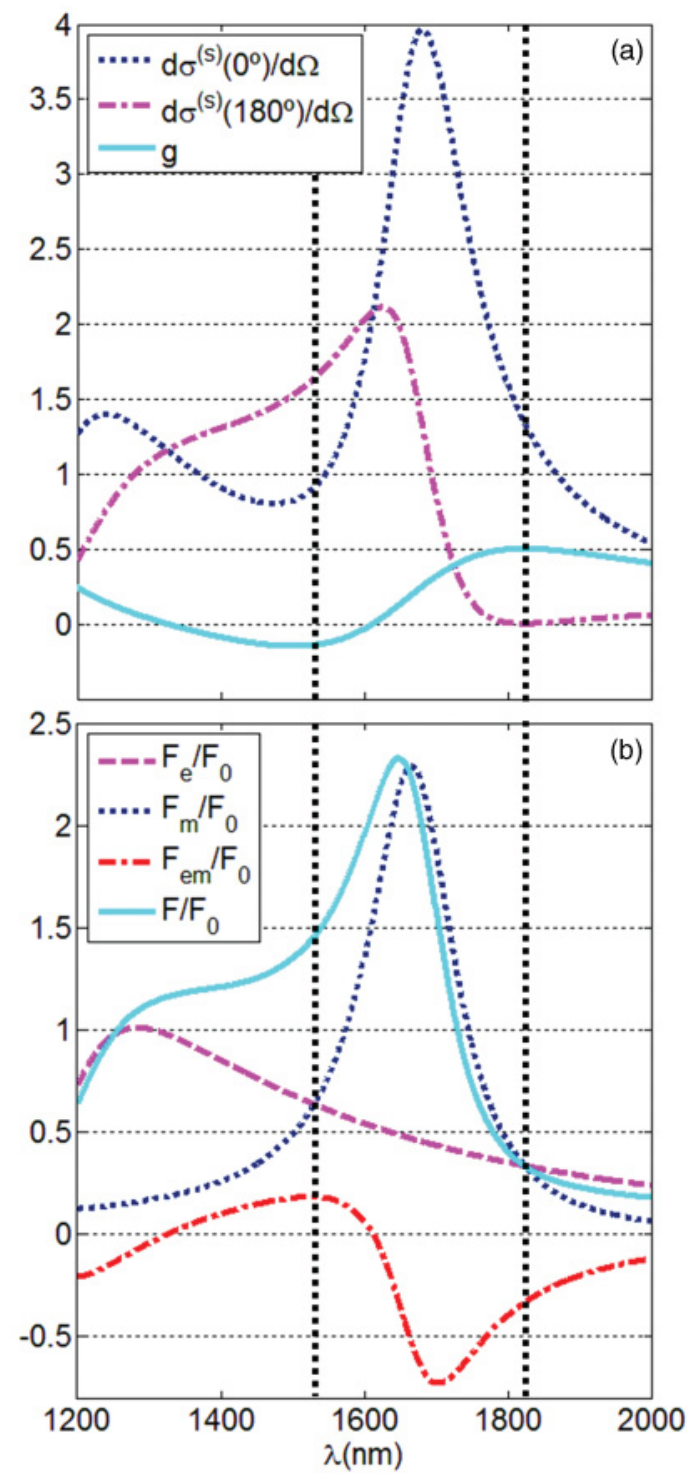

FIG. 2. (Color online) (a) Forward and backward differential scattering cross sections and asymmetry factor versus the wavelength for a silicon spherical particle of radius $a=230 \mathrm{~nm}$ and $\epsilon=12$. (b) Different contributions to the total radiation pressure versus the wavelength for the same particle. Normalization is done by $F_{0}=4 \pi a^{3} k\left|\mathbf{E}_{0}\right|^{2} / 2$. The vertical lines mark, from right to left, the first and second Kerker conditions. 
must always be positive, as required from causality [11]. In the quasistatic approximation, they produce zero-forward scattered power [22]) with an asymmetry factor $g \approx-1 / 2$, which means that $l^{*} \approx(2 / 3) l_{s}$ [note that strong backscattering would correspond to $g=-1$ and $\left.l^{*}=(1 / 2) l_{s}\right]$. Thus, for a dilute suspension of arbitrary nonabsorbing dipolar particles, Eq. (5) and the Kerker conditions impose the following limits to the MFP:

$$
0.66 \ell_{s} \lesssim \ell^{*} \leqslant 2 \ell_{s} .
$$

While the results above are of general application, it is interesting to discuss two specific examples that could be realized experimentally. As a direct consequence of the discussion above, perfectly conducting dipolar spheres [25], for which $\alpha_{e}^{(0)}=-2 \alpha_{m}^{(0)}$, have an asymmetry factor that is negative. In particular in the quasistatic limit this factor becomes $g=-0.4$, which implies $\ell^{*} \approx 0.7 \ell_{s}$ for a random dispersion of small, perfect conducting spheres. This result may be especially relevant in the terahertz regime where metals can often be considered as perfect conductors [26].

Silicon spheres with radius $a=230 \mathrm{~nm}$ have been proven to behave as dipolar magnetodielectric particles with a strong magnetic dipole response in the near infrared region. In Fig. 2 we show the forward and backward differential scattering cross sections and the asymmetry factor $g$ as well as the variation of $\left\langle\mathbf{F}_{e-m}\right\rangle,\left\langle\mathbf{F}_{e}\right\rangle$, and $\left\langle\mathbf{F}_{m}\right\rangle$ for a Si sphere of radius $230 \mathrm{~nm}$. Notice when the first Kerker condition is fulfilled, $g=1 / 2$ and $\frac{d \sigma^{(s)}}{d \Omega}\left(180^{\circ}\right)=0$ [Fig. 2(a)] and $F_{e}=F_{m}=-F_{e-m}=F$ [Fig. 2(b)].

Nevertheless, if one imposes restrictions on $\alpha_{e}$ and $\alpha_{m}$, then other situations appear. From Eq. (6) one can see that $g$ is maximal and equal to $1 / 2$ where $\frac{d \sigma_{s}}{d \Omega}\left(180^{\circ}\right)$ is zero and it has a minimum value at a wavelength where $\frac{d \sigma_{s}}{d \Omega}\left(0^{\circ}\right)$ is minimal. This minimum value of $g$ is also negative if $\frac{d \sigma_{s}}{d \Omega}\left(0^{\circ}\right)<\frac{d \sigma_{s}}{d \Omega}\left(180^{\circ}\right)$. This is illustrated in the case of the above-mentioned $\mathrm{Si}$ sphere of radius $230 \mathrm{~nm}$. As seen in Fig. 2(a), at $\lambda=1530 \mathrm{~nm} g$ has a minimum equal to -0.15 , which corresponds to the minimum forward DSCS, whereas where the first Kerker condition holds, when the backscattering cross section is zero, the $g$ factor has a maximum equal to $1 / 2$.

In conclusion, we have demonstrated that, surprisingly and without further assumptions about collective interactions, dilute suspensions of dipolar semiconductor spheres, e.g., $\mathrm{Si}$ and $\mathrm{Ge}$, have an optical frequency range in which their scattering asymmetry parameter is negative and hence they acquire a transport MFP smaller than their scattering MFP. This is made possible by the magnetodielectric nature of these particles and the consequent electric-magnetic dipole interference, which in addition leads to a simple relation between the electric-magnetic interaction photonic force and the asymmetry factor. This also applies to perfectly conducting spheres at longer wavelengths.

This work was supported by the Spanish MEC through Grants No. FIS2009-13430-C01-C02 and No. CSD200700046 (Consolider NanoLight) and by the Comunidad de Madrid Microseres Project No. S2009/TIC-1476. M.Y. thanks the Mexican Consejo Nacional de Ciencia y Tecnología for a postdoctoral grant (No. 000000000162768). F.S. acknowledges financial support by the Swiss National Science Foundation (Project No. 132736) and the Adolphe Merkle Foundation.
[1] Waves and Imaging through Complex Media, edited by P. Sebbah (Kluwer Academic, Dordrecht, 2001); in Wave Scattering in Complex Media: From Theory to Applications, edited by B. A. van Tiggelen and S. E. Skipetrov, NATO Science Series II: Mathematics, Physics and Chemistry, Vol. 107 (Kluwer Academic, Dordrecht, 2003).

[2] A. Yodh and B. Chance, Phys. Today 48(3), 34 (1995); S. K. Gayen and R. R. Alfano, Opt. Photon. News 7, 17 (1996); J. Ripoll, V. Ntziachristos, J. P. Culver, D. N. Pattanayak, A. G. Yodh, and M. Nieto-Vesperinas, J. Opt. Soc. Am. A 18, 821 (2001).

[3] D. A. Weitz and D. J. Pine, in Dynamic Light Scattering, edited by W. Brown (Oxford University Press, New York, 1993); G. Maret and P. E. Wolf, Z. Phys. B 65, 409 (1987); D. J. Pine, D. A. Weitz, P. M. Chaikin, and E. Herbolzheimer, Phys. Rev. Lett. 60, 1134 (1988); R. Lenke and G. Maret, in Multiple Scattering of Light: Coherent Backscattering and Transmission, edited by W. Brown (Gordon \& Breach, Reading, UK, 2000).

[4] F. Scheffold and P. Schurtenberger, Soft Mater. 1, 139 (2003).

[5] A. Derode, A. Tourin, J. de Rosny, M. Tanter, S. Yon, and M. Fink, Phys. Rev. Lett. 90, 014301 (2003); S. H. Simon, A. L. Moustakas, M. Stoytchev, and H. Safar, Phys. Today 54(9), 38 (2001); G. Lerosey, J. de Rosny, A. Tourin, and M. Fink, Science 315, 1120 (2007).
[6] H. C. Van de Hulst, Light Scattering by Small Particles (Wiley, New York, 1957).

[7] C. F. Bohren and D. R. Huffman, Absorption and Scattering of Light by Small Particles (Wiley, New York, 1983).

[8] F. Pinheiro, A. Martinez, and L. Sampaio, Phys. Rev. Lett. 84, 1435 (2000).

[9] A. García-Etxarri, R. Gómez-Medina, L. S. Froufe-Pérez, C. López, L. Chantada, F. Scheffold, J. Aizpurua, M. Nieto-Vesperinas, and J. J. Sáenz, Opt. Express 19, 4815 (2011).

[10] M. Nieto-Vesperinas, J. J. Sáenz, R. Gómez-Medina, and L. Chantada, Opt. Express 18, 11428 (2010).

[11] M. Nieto-Vesperinas, R. Gómez-Medina, and J. J. Sáenz, J. Opt. Soc. Am. A 28, 54 (2011); R. Gómez-Medina, M. Nieto-Vesperinas, and J. J. Sáenz, Phys. Rev. A 83, 033825 (2011).

[12] R. Gómez-Medina, B. García-Cámara, I. Suárez-Lacalle, F. González, F. Moreno, M. Nieto-Vesperinas, and J. J. Sáenz, J. Nanophoton. 5, 053512 (2011).

[13] S. Fraden and G. Maret, Phys. Rev. Lett. 65, 512 (1990).

[14] For the wavelength dependence of $\ell_{s}$ in disordered photonic crystals see P. D. García, R. Sapienza, L. S. Froufe-Pérez, and C. López, Phys. Rev. B 79, 241109(R) (2009).

[15] J. M. Ziman, Philos. Mag. B 6, 1013 (1961); N. W. Ashcroft and J. Lekner, Phys. Rev. 145, 83 (1966). 
[16] R. W. Hartand and R. A. Farrell, J. Opt. Soc. Am. 59, 766 (1969); G. B. Benedek, Appl. Opt. 10, 459 (1971).

[17] L. F. Rojas-Ochoa, J. M. Méndez-Alcaraz, J. J. Sáenz, P. Schurtenberger, and F. Scheffold, Phys. Rev. Lett. 93, 073903 (2004); R. Pierrat, N. B. Braham, L. F. Rojas-Ochoa, R. Carminati, and F. Scheffold, Opt. Commun. 281, 18 (2008).

[18] M. Reufer, L. F. Rojas-Ochoa, S. Eiden, J. J. Sáenz, and F. Scheffold, Appl. Phys. Lett. 91, 171904 (2007); P. D. García, R. Sapienza, A. Blanco, and C. López, Adv. Mater. 19, 2597 (2007).

[19] This wavelength dependence of the transport mean free path, induced by short-range order, is also the origin of the structural colors of blue bird feather barbs as discussed by R. O. Prum,
R. H. Torres, S. Williamson, and J. Dyck, Nature (London) 396, 28 (1998); Liew et al., Opt. Express 19, 8208 (2011).

[20] X.-D. Liu, L.-T. Hou, H.-T. Wang, S.-G. Li, and G.-L. Huo, Chin. Phys. Lett. 19, 1353 (2002).

[21] P. C. Chaumet and A. Rahmani, Opt. Express 17, 2224 (2009).

[22] M. Kerker, D. S. Wang, and C. L. Giles, J. Opt. Soc. Am. 73, 765 (1983).

[23] B. García-Camara, F. Moreno, F. Gonzalez, and J. M. Saiz, J. Opt. Soc. Am. A 25, 2875 (2008).

[24] W. M. Irvine, J. Opt. Soc. Am. A 55, 16 (1965).

[25] J. D. Jackson, Classical Electrodynamics, 3rd ed. (Wiley, New York, 1998).

[26] K. Song and P. Mazumder, IEEE Trans. Electron. Devices 56, 2792 (2009). 